\title{
Energy Agents - Foundation for Open Future Energy Grids
}

\author{
Christian Derksen \\ DAWIS - University of Duisburg- \\ Essen, Schützenbahn 70, 45127 \\ Essen, Germany \\ Email: \\ christian.derksen@icb.uni-due.de
}

\author{
Rainer Unland \\ DAWIS - University of Duisburg- \\ Essen, Schützenbahn 70, 45127 \\ Essen, Germany \\ Email: \\ rainer.unland@icb.uni-due.de
}

\begin{abstract}
Redefining our energy landscape by means of regenerative, volatile and decentralized organized systems represents a major challenge for the creation of distributed control solutions. Standards are required that permit describing the variety of energy conversion systems and that enable an interoperable exchange of energy amounts in an open, flexible manner by concurrently taking into account technical and market requirements. This paper introduces the concept of unifying Energy Agents. They have the potential to reduce the ever growing complexity that comes along with the various solutions presented or that are already available at the market. In contrast to that, the notion of Energy Agents stands in our view as a representative for a required methodology that enables a consistent development of de-centralized control solutions. In order to demonstrate this approach, the application of Energy Agents in a smart house scenario is discussed. It is shown how arbitrary agents with different levels of sophistication and abilities can cooperate with each other in a smooth way. It is our strong believe that a stepwise and standardized development of Energy Agents representing the needed decentralized control solutions is needed for a sustainable design of an open Future Energy Grid.
\end{abstract}

\section{INTRODUCTION}

$\mathrm{R}$ eflecting the tendencies for more decentralized controlled energy conversion systems and the further increasing number of IT-enriched smart systems in general, a picture can be drawn, that describes the future energy landscape as a complex, globally connected and mainly software driven system. Smart Markets need to build on top of an underlying technical systems with inherent flexibility that guarantees a stable volatile energy production [1], in order to reach climate targets [2] or just to maximize organizational profit [3]. However, global goals, such as the stabilization of a distribution networks, require a minimum of adaptive interoperability that has to be expressed in one standard. We believe that the developments in Smart Grids and related areas are now at a point, were it has to be asked, if we want to build control systems that are creating new monopolies, caused by proprietary software solutions, or if we want to create an unbundled and open energy supply that, on the one hand, offers the needed intelligent flexibility and, on the other hand, strongly supports further developments over the next decades? Assuming that the latter is the case, it is obvious that software standards are required that prevent our with respect to technical basics and market regulations already highly complex energy supply from becoming even more complex and possibly uncontrollable.

With the concept of unified Energy Agents and its associated frameworks and tools that are a unifying Energy Option Model (EOM) [4] and the agent execution environment Agent.GUI [5], we provide a development and validation environment that allows a systematic and stepwise progression for decentralized control solutions. We believe that a stepwise definition of concrete use case scenarios and a clear definition of the actual tasks and capabilities of the on-site software - that are in our view Energy Agents - are strongly required. Corresponding to these different scenarios, Energy Agents have to be defined with specific levels of sophistication that have to be validated through simulations and in test-bed applications before they can be applied in real on-site systems. Furthermore, energy carrier like natural gas, heat and other have to be considered in order to define a comprehensive problem description that reflects the complex and interconnected nature of the real energy supply.

This paper presents an application example of our Energy Agent approach. For this, the next section provides the theoretical background. This will be followed by the presentation of the concept of Energy Agents. Section 4 presents their application in a smart house scenario. A discussion and an outlook will conclude the paper.

\section{II.BACKGROUND}

Agents and Multi-Agent systems (MAS) are already used by a large scientific as well as industrial community due to their inherent ability to naturally describe the distributed problems and scenarios that comes along with the energy domain. A significant number of papers already described successful applications of agents in specific Smart Grid scenarios, as for example in virtual power plants [6], in Demand-Side-Management systems [7] or within pricebased, indirect controlled approaches that are known as Demand Response [8]. Despite these promising first 
applications connecting IT-concepts and standards are still missing that enable a large-scale rollout of Smart Grid solutions by concurrently providing a meaningful function and an investment security for end users and producers. The problem to be tackled here is a missing open architecture. Right now there is the danger of new monopolies, caused by investment decisions towards proprietary Smart Grid solutions that may preclude customers from switching to a new energy provider. Another threat of an uncontrolled growth of arbitrary (software) solutions is the resulting complexity and a possible in-deterministic behavior of the overall system, especially if every software component in a Future Energy Grid can behave as it likes.

Such types of problems have already been discussed in the scientific community that generally has been studying the building of agent organizations and the related topics of reputation and trust [9]. Following [10], MAS-organizations can roughly be categorized by two dimensions. The first dimension permits to differentiate between MAS that rely on existing parent organizational structures or do not. This classifies designs of MAS into so called "agent-centered" or "organization-centered" designs. The second dimension focusses on the abilities of agents. It distinguishes by whether they have organizational knowledge or not. Especially the latter dimension is to be equated with the question if an agent organization represents a concept that only serves the static design of a multi-agent system or if the organizational affiliation is relevant at the runtime of the individual agents. Table 1 below shows the resulting types of agent organizations derived from these two differentiations.

Table 1: Classification of Agent ORganizations [11]

\begin{tabular}{|c|c|c|}
\cline { 2 - 3 } \multicolumn{1}{c|}{} & $\begin{array}{c}\text { Agents without } \\
\text { organizational } \\
\text { knowledge }\end{array}$ & $\begin{array}{c}\text { Agents with } \\
\text { organizational } \\
\text { knowledge }\end{array}$ \\
\hline $\begin{array}{c}\text { Agent-centered Design } \\
\text { (no given formal } \\
\text { organizational structure) }\end{array}$ & I. & II. \\
\hline $\begin{array}{c}\text { Organization-centered } \\
\text { design (given formal } \\
\text { organizational structure) }\end{array}$ & III. & IV. \\
\hline
\end{tabular}

Without discussing every type of agent organization in detail here, we would like to point out that in quadrant IV. open agent organizations are located, where agents are able to dynamically decide if they want to join or leave an agent organization. To support functionalities like this, further efforts has to be spent in order to enable such dynamic and thus adaptive behavior. We believe that this is - to some extent - indispensable for the further systematic development of an open, IT-controlled Future Energy Grid. Consequently, this requires commitments and standards that focus on the local software components on-site that we propose to call Energy Agents.

\section{ENERGY AGENTS}

The concept of Energy Agents was first introduced by [12]. There an Energy Agent was defined as a representative of a technical system - or better an energy conversion process - that acts on an operational level and that will not replace but extend on-site controllers. Thus, Energy Agents can be seen as additional software artefacts, capable to autonomously manage the capacitive abilities of associated technical systems in terms of energy production and usage and on behalf of the owner or other stakeholders. According to the tasks assigned to it, the inherent complexity and thus the sophistication or so called Integration Level (IL) of an Energy Agent may differ; different tasks, like monitoring, learning or constraint satisfying planning and optimizations are conceivable in this context.

Furthermore, an approach to validate decentralized control systems was proposed by means of a systematic development process that includes simulations and test bedapplications before an on-site hard- and software usage should be considered. By implementing exchangeable behaviors for simulated or real world interactions between agents and local system controllers, the main software components of the Energy Agent are to be preserved in regard to the development process. Finally, a concept for a generalized option, cost and action model was introduced that is capable of describing possible and current operation phases of technical systems and that serves the Energy Agents as a unified base model for reasoning processes. In these considerations also hybrid energy systems and infrastructures were taken into account, as for example natural gas or heating systems.

Figure 1 below gives an impression and compares two types of Energy Agents, equipped with different capabilities. It is shown that the shell that represents the Energy Agent is coupled in two ways to the outside world. First, by means a connection to a technical system and second by it capabilities for inter-agent communication. Here the coupling to the technical system can be realized in various ways; e.g. by means of well-known protocols like IEC61850, the Common Interface Modell (CIM), OPC UA and other.

Following our proposed gradation of integration levels (see reference above), both Energy Agents have a domain specific model - the Energy Option Model (EOM) - that describes the operating capabilities and the scope for possible actions of the underlying technical system. Both agents can be located in IL3, as both have the ability to (re-) act on external signals or information in general. Beside that they have the ability to monitor the underlying technical system and provide information that help to predict the systems behavior in regard to its energy production or usage, which is recommended by the introduced lower integration level IL0 - IL2. 

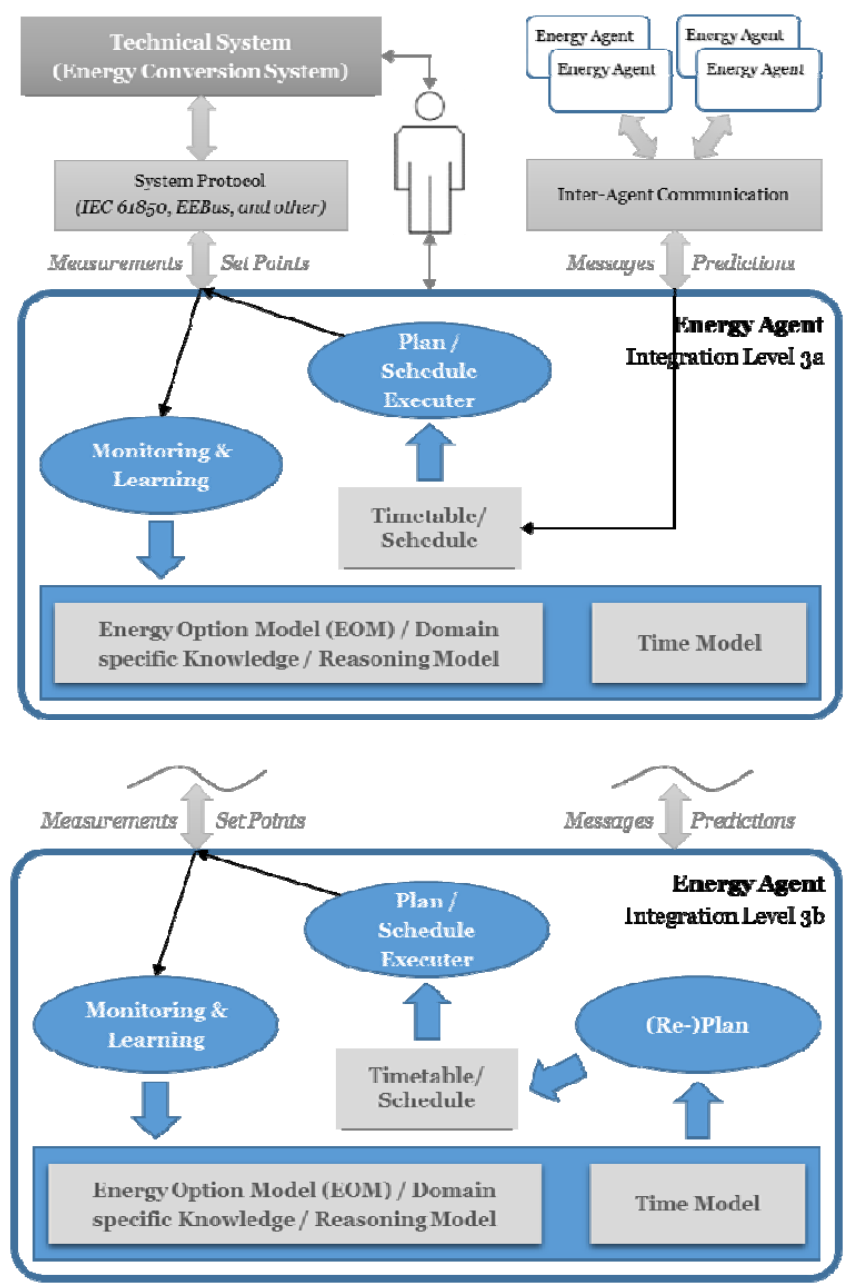

Fig.1: Structure and functionalities of Energy Agents with different Integration Level

Furthermore, the picture underlines that in regard to the requirements for an open Future Energy Grid the definition of the actual application scenario is absolutely indispensable, since the functionalities that are to be implemented within an Energy Agent strongly depends on these scenarios. Even both Energy Agents are located in IL3, the upper agent just receives and accepts schedule from a superordinate control unit, while the bottom Energy Agent uses the EOM in order to determine an optimized schedule by itself.

With the Energy Agents above, two different Smart Grid approaches are described that have often be used and discussed in scientific publications. While the first Energy Agent could be applied in a Demand Side Management scenario, where a central process decides for operational states and set-point configurations, the second can be used within a more decentralized Demand Response approach, e.g. reacting on external price signals.

It is crucial to realize that in both cases the Energy Option Model, purposed with the Energy Agent approach, forms the base for both application cases. With its approach that allows to uniformly describe energy conversion systems, the EOM allows to transfer and relocate decision making processes and thus to realize different types of control solutions; either centralized or decentralized solutions. We believe that such unified descriptions is the first step towards the realization of an open Future Energy Grid, since it allows an adaptive management of net-coupled technical systems or energy conversion processes. As a second step, the application scenario with its corresponding rules, policies and behaviors have to be standardized, so that developer of Smart Grid solutions have not only requirements but also degrees of freedom for their developments. Therefore, the integration levels proposed have to be fully specified, while the levels needs to be developed and extend over the next years.

\section{APPLICATION OF ENERGY AgENTS}

With the application scenarios presented here, we demonstrate different level of sophistication for Energy Agents by means of comparing Demand Side Management with a price-based Demand Response approach. As use case we selected a smart house, equipped with different technical systems that are each "interfaced" by an Energy Agent.

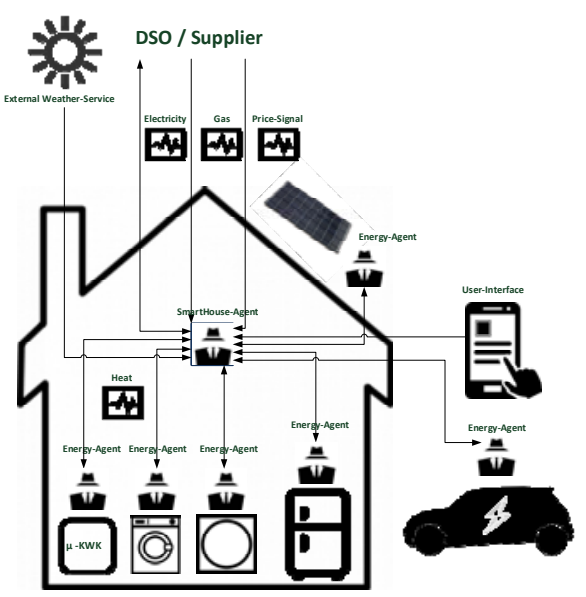

Fig.2: Smart-House Scenario as use case for Energy Agents

As the figure shows, all considered devices are equipped with Energy Agents that include a corresponding EOM for the specific technical system. A concrete example of a single EOM will be shown further below with an Electrical Vehicle.

In detail the dryer and the washing machine were modelled as "smart" batch processes. Such process allows to shift the actual start of the selected program by staying in a waiting operating state. Knowing the energy consumption of the device that is given by measurements of previous runs, the energy usage can be shifted into a designated time range.

The fridge was modelled as repetitive system whose main task is to hold the inner temperature within a range of 4 $6^{\circ} \mathrm{C}$. By varying these temperatures, using them as set-points for the internal controller of the fridge, the Energy Agent is able to increase or decrease these temperatures by $\pm 1{ }^{\circ} \mathrm{C}$, in order to flexibilize the energy consumption. 
Since the energy production of the photovoltaic plant basically depends on the solar radiation that occurs at the specific day, the power production was just statically considered for the experimental setup. Needed information were taken from historically data for the specific day. In a more elaborated setup the EOM could also be used in order to calculate energy production based on weather information that could be determined by forecasts.

For the $\mu \mathrm{CHP}$, the manufacturer information of a Vaillant $\mu$ CHP ecoPowerl.0 was modelled with EOM. Here four operating states were defined that are a standby, an acceleration phase, a normal operation and a shutdown that is followed by the standby of the system again.

In the case of the Demand Side Management scenario the Smart House Agent plays the central role in all optimization issues. It collects and processes all relevant information from every subordinated Energy Agent, like all individual EOMs as well as the actual states of the systems. Thus, the Smart House Agent acts as an aggregator for the whole house. With the aim to optimize energy costs, especially also by first using as much energy as possible from the local energy sources, the optimization approach is carried out for a whole day. In the Demand Response scenario the role of the Smart House Agent was restricted and simplified. Here it basically forwarded external price signals or needed external information, like the outside temperature, needed as forecast from an external weather service. The actual optimization approaches were implemented in each of the Energy Agents.

To demonstrate the efforts in defining a system specific EOM, Figure 3 below shows one exemplary Energy Option Model for the electrical vehicle (EV) used.

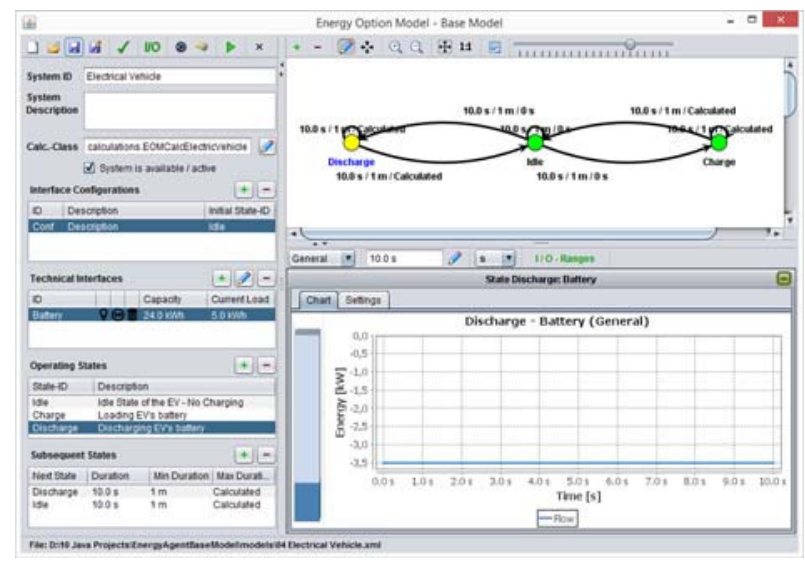

Fig.3: Base Model of an Electrical Vehicle as example

In general, a single technical system is described by the connections to an energy carrier-dependent network that are described by Interface Configurations and Technical Interfaces to the corresponding network (e.g. an EV can differently be connected to an electrical network). If a storage is available, as this is the case for an EV, a system can be defined with one storage per energy carrier. The Image above shows as an example that the used EV has a storage capacity of $24 \mathrm{kWh}$ and a current load of $5 \mathrm{kWh}$.
Beyond that, the image shows the graph of the possible operating states for the EV and possible subsequent states. Here, the operating states Idle, Charge and Discharge are defined. Additionally, for each of this state the actual energy flow are defined, as for example the $3.5 \mathrm{~kW}$ electrical output when discharging the EV battery. Figure 4 below shows the settings for the evaluation of a single technical system.

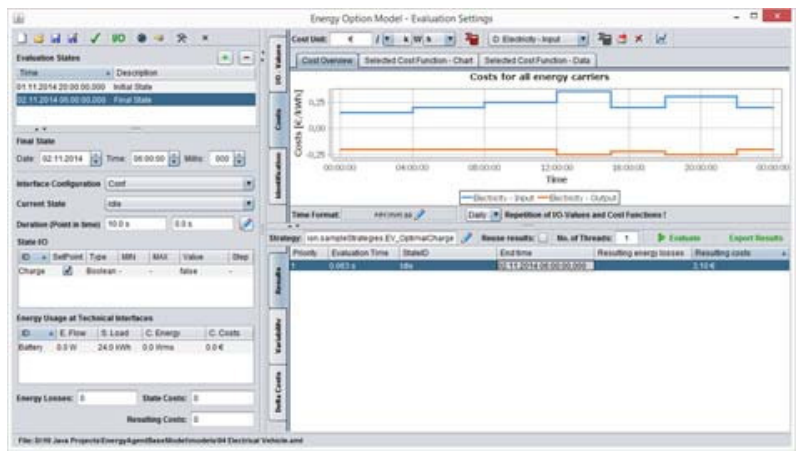

Fig.4: Evaluation settings and result for a single technical system

On the left hand side, the current and the wished technical system states can be defined for the evaluation period. Here the EV was connected to the network at 8 p.m., while the charging process should be finished at the next morning at 6 a.m.; the wished storage load was set to the maximum storage capacity of $24 \mathrm{kWh}$. On the right side above, the specific cost functions for each energy carrier and for a specific direction (input or output) can defined. It can be seen that the consumption is defined with positive cost values, while the production or feed-in is defined with negative cost values. Developing an individual evaluation strategy a tailored algorithm can be developed and used in order to produce an optimised schedule for the actual technical system. Results can look like the charts shown in Figure 5, where the storage load and the costs over time are presented. In fact, the evaluation strategy used has the aim to cost optimally charge the EV. For this a quite simple algorithm was developed that uses the cost information (shown in Figure 4) and the demand of electrical energy that is given by the goal state within the specified time range. By a simple search of the most inexpensive time ranges for the charging operating state and a Greedy based decision process, the cost optimal schedule for the EV could easily be determined.
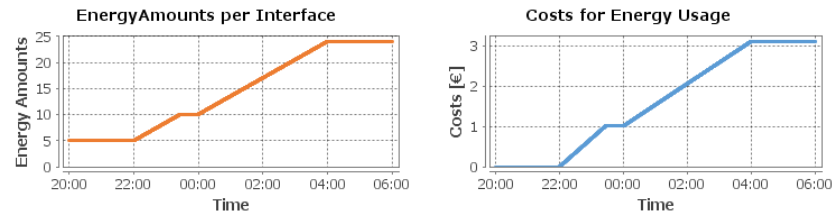

Fig.5: Cost optimized charging of an Electrical Vehicle

As general approach for an evaluation or the optimization of a single technical system, the framework of the EOM provides a graph-based decision system. Based on the time discretization of operational states, the decision system 
allows to fully describe technical systems states in regard to (possibly predicted) input and output information, as well as to energy flows at network interfaces. Further, storage loads, transferred energy amounts, energy losses and price signals can be considered for the preparation of a schedule, while the actual optimization problem can individually differ. Figure 6 below shows the so called differences graph of this approach.

System State: global time, duration, set-points, measurements, energy flows and storage load

Change of State: energy flows, amounts and losses, as well as resulting costs (connection-specific and in total)

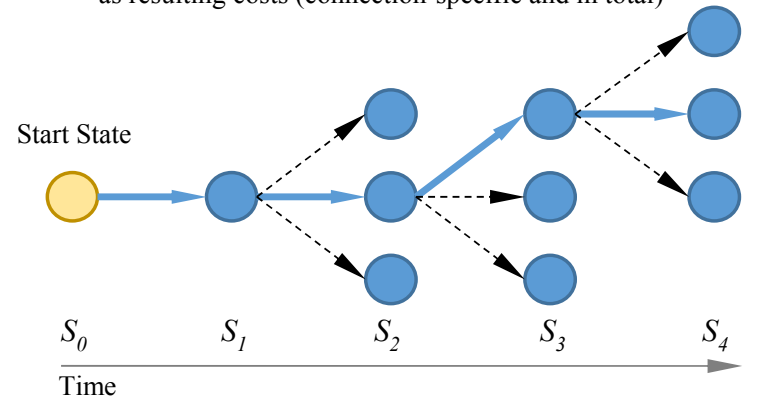

Fig.6: Differences graph for evaluation strategies

The graph will first be created by the current system state $S_{0}$ that is defined with the start state for the evaluation process. Since an operating state that is defined by the base model of the EOM describes also the time range for this state, the subsequent state $S_{I}$ can be determined with the end of this operating state. With this, energy flows per interface and energy losses that were transferred or produced within this period, as well as the new storage load and thereto corresponding costs will be calculated. After that, and derived from the graph of possible operating states that is defined with the base model of the EOM, the possible subsequent operating states can be determined over time. In order to decide which system states is to be used for a schedule, a decision must be taken at each of the nodes defined by the differences graph. To support informed decisions, the framework of the EOM provides the information mentioned above (energy flows etc.) as precalculated set to the decision making process that is located within an individual strategy for an evaluation. Since each technical system might require different background information for such decision processes, the framework of the EOM provides an adaptive programming interface that is named as (Abstract) Evaluation Strategy and that allows to create individual solutions.

The aggregation method for several technical systems that is provided by the EOM and that was used within the Smart House Agent is realized in the sense of a system of systems. Analogously to a single technical system, the aggregator will first be considered as a technical system, summarizing the sub systems in regard to interfaces by energy carrier and by add up storage capacities. In more complex scenarios, were wider areas than a house are to be considered, an aggregation may additionally require a network calculation.
Similar to the Evaluation Strategy for single technical systems, a strategy for aggregated technical systems can individually be designed. In contrast to single systems, decisions must be made for each subordinate system, so that several decision graphs will concurrently be used during an evaluation of aggregated technical systems.

What was shown in the illustrations above as user dialog can also be used "head-less" within an Energy Agent. In this case it is the task of an Energy Agent to get and provide the needed base information for a single technical system or an aggregation of technical systems (e.g. cost information) and start an appropriate evaluation process, if required.

For the centrally controlled Smart House example, a first heuristic approach was chosen for the decision making process that discretized again the assessment parameter that in turn corresponded to the optimization goal: the price for energy. More concrete: a high price was rated with lowest priority, while a low price was rated with a high priority. Since we assumed a perfect prediction provided by the EOM's of the technical systems, no spontaneous re-planning was intended in this scenario. However, we are aware that a re-planning would be necessary if any of the subordinated systems deviates from its prediction. Nevertheless, the state and solution space for the Smart House scenario was already huge and could easily exceed the available computing capacities. The application of the heuristic approach, however, reduced the search and solutions space substantially and with it the time to calculate a result.

Figure 7 below shows the cost functions that were used for the assessment of the energy usage, while Figure 8 presents - as an example - the resulting electrical power flow for the aggregated Smart House scenario.

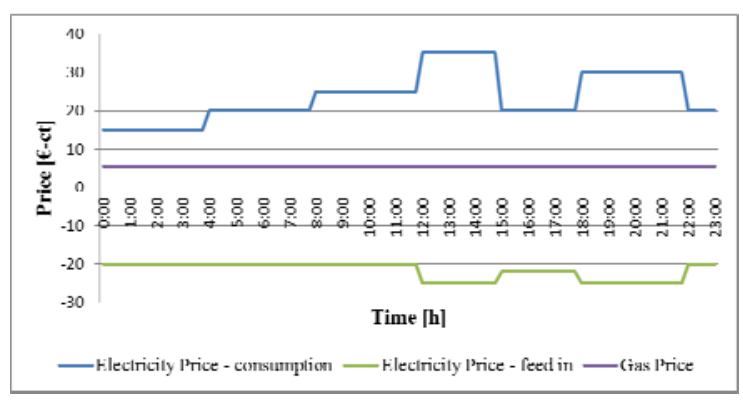

Fig.7: Costs and Revenue Functions by Energy Carrier

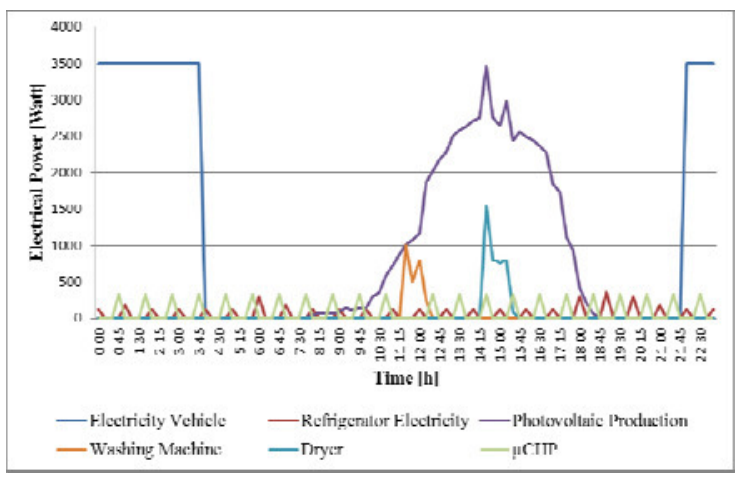

Fig.8: Electrical Power Usage for the aggregated Smart House 
Due to the mobility phase, the electrical vehicle was not connected during daytime. Hence, it was charged by night using the cheapest prices from 10 p.m. until approx. 4 a.m. in the morning. The electrical power usage for the washing machine and the tumble dryer was shifted into the power production phase of the photovoltaic plant. Since the $\mu$ CHP and the fridge are repetitive working systems, these system are providing just small flexibility ranges. It is a task for the future work to improve our approach in order to optimize our first approach.

Overall, it was found that the overall energy costs could be reduced by using the aggregation case and comparing it to the Demand Response is possible. Since in an aggregation the interdependencies of several technical systems can be considered, this result was not surprising us. However, the complexity which we had already to face was.

Much more important for us was, however, to find a way to adaptively describe and connect hybrid technical systems by using the approach of the unifying Energy Option Model. Based on that, more sophisticated optimization approaches will be developed and tested in the future, as well as the number of technical systems considered can hopefully be diversified and increased. Since the developed Evaluation Strategies are based on a graph, several meta-heuristic approaches are conceivable here, as for example ant-based approaches and other.

\section{V.Discussion, OUTLOOK \& CONCLUSION}

This paper has presented the concept and the application of Energy Agents that are equipped with a unifying Energy Option Model. Since this model has the capability to describe all relevant types of energy conversion processes, it provides the foundation for the design of new adaptive methods that permit a dynamic aggregation and optimization of groups of technical systems. As a proof of concept an application scenario for a Smart House was presented that compares a demand response case with a case where a Demand Side Management for the house was realized. Based on the Energy Option Model and Agent.GUI, that is already freely available as open source, it is our intention to provide several predefined scenarios as the here presented Smart House scenario in the future.

Moreover, the concept of the Energy Agent will be improved and developed under the project Agent.HyGrid in the next years [13]. Here it is planned to close the gap between agent-based simulations and real-world applications in order to produce comparable results for both cases. Thus, a realistic laboratory and test-bed environment will be created for decentralized control solution of Future Energy Grids.

Overall, we believe that urgently standards are required that enable such homogeneous and in particular open developments; for science and for systems used in real applications on-site. We further believe that therefore the concept of a generally accepted Energy Agent with a unified description of the underlying technical system that we call Energy Option Model is the necessary foundation. Together with a refined and commonly accepted concept of an Energy Agent an open Future Energy Grid could be designed that provides the perfect environment for further elaborations and improvements over the next decades. We further believe that such standardization is absolutely indispensable in order to reach the overall objectives, associated with the energy transition or with the planned European Energy Union.

\section{REFERENCES}

[1] C. Oerter and N. Neusel-Lange, "LV-grid automation system - A technology review," in PES General Meeting | Conference Exposition, 2014 IEEE, 2014, pp. 1-5.

[2] E. Bernier, F. Maréchal, and R. Samson, "Multi-objective design optimization of a natural gas-combined cycle with carbon dioxide capture in a life cycle perspective," Energy, vol. 35, no. 2, pp. 1121$1128,2010$.

[3] T. Linnenberg, I. Wior, S. Schreiber, and A. Fay, "A market-based multi-agent-system for decentralized power and grid control," in Emerging Technologies Factory Automation (ETFA), 2011 IEEE 16th Conference on, 2011, pp. 1-8.

[4] C. Derksen and R. Unland, "Hybrid Energy Option Models for Unified Energy Agents," in Operations Research, Aachen, Germany, 2014.

[5] C. Derksen and R. Unland, "An advanced agent-based simulation toolbox for the comprehensive simulation of future energy networks," in Smart Grid Technology, Economics and Policies (SG-TEP), 2012 International Conference on, 2012, pp. 1-4.

[6] D. Nestle and J. Ringelstein, "Integration of DER into Distribution Grid Operation and Decentralized Energy Management," Smart Grids Europe, vol. 19, 2009.

[7] [Online] Available at: http://www.e-energy.de/de/modellregionen.php.

[8] A. Faruqui and S. Sergici, "Household response to dynamic pricing of electricity: a survey of 15 experiments," Journal of Regulatory Economics, vol. 38, no. 2, pp. 193-225, 2010.

[9] I. Pinyol and J. Sabater-Mir, "Computational trust and reputation models for open multi-agent systems: a review," Artificial Intelligence Review, vol. 40, no. 1, pp. 1-25, 2013.

[10] O. Boissier, J. F. Hübner, and J. Simao Sichman, "Organization Oriented Programming: From Closed to Open Organizations," in Engineering Societies in the Agents World VII, vol. 4457, G. M. P. O’Hare, A. Ricci, M. O'Grady, and O. Dikenelli, Eds. Springer Berlin Heidelberg, 2007, pp. 86-105.

[11] M. Wester-Ebbinghaus, "Von Multiagentensystemen zu Multiorganisationssystemen - Modellierung auf Basis von Petrinetzen," Universität Hamburg, Von-Melle-Park 3, 20146 Hamburg, 2010.

[12] C. Derksen, T. Linnenberg, R. Unland, and A. Fay, "Unified Energy Agents as a Base for the Systematic Development of Future Energy Grids.," in MATES, 2013, vol. 8076, pp. 236-249.

[13] C. Derksen, T. Linnenberg, N. Neusel-Lange, and M. Stiegler, "Agent.HyGrid: A seamless Development Process for agent-based Control Solutions in hybrid Energy Infrastructures," in SmartER Europe - E-world energy \& water, Essen, Germany, 2015. 